PROCEEDINGS OF THE

AMERICAN MATHEMATICAL SOCIETY

Volume 126, Number 6, June 1998, Pages 1583-1596

S 0002-9939(98)04183-5

\title{
POLYNOMIAL EXPANSION
}

\author{
SHREERAM S. ABHYANKAR
}

(Communicated by Wolmer V. Vasconcelos)

Dedicated to Herbert Popp for his Sixtieth Birthday

\begin{abstract}
The idea of initial form is refined into the concept of polyform.
\end{abstract} This can be used to streamline desingularization.

\section{SECTION 1: InTRODUCTION}

We shall refine the idea of the initial form of an element in a regular local ring by introducing the concept of its polyform. The existence of polyform will be proved relative to any finite sequence in any (commutative unitary nonnull) ring satisfying some mild separation conditions. For uniqueness, the sequence will be assumed to be regular. The concept of polyform is expected to simplify desingularization proofs and related matters. In effect, many desingularization processes amount to finding a regular system of parameters relative to which the polyform of a given element in a regular local ring has a tight shape. We shall illustrate this by revisiting desingularization of plane curves in any characteristic including mixed characteristic. What we shall reprove is that, given any curve on any nonsingular algebraic or arithmetic surface, by applying a finite sequence of quadratic transformations to the surface it can be achieved that the total transform of the curve has only normal crossings. This will be a simplified version of the canonical desingularization of curves described in $[\mathrm{AbhD}]$. Similarly, in a later communication, we hope to use polyforms to give a simplified version of the higher dimensional canonical desingularization processes discussed in $[\mathrm{AbhG}]$ and $[\mathrm{AbhW}]$.

\section{SeCtion 2: PolyeXPAnsion AND POLYForm}

Let $I$ be the ideal generated by a finite sequence $x=\left(x_{1}, \ldots, x_{m}\right)$ in a ring $R$. As usual, let $N$ be the set of all nonnegative integers, and let $N^{m}$ be the set of all $m$-tuples $a=\left(a_{1}, \ldots, a_{m}\right)$ of nonnegative integers; the set $N^{m}$ is partially ordered: $a \leq a^{\prime}$ means $a_{i} \leq a_{i}^{\prime}$ for $1 \leq i \leq m$. Let $X=\left(X_{1}, \ldots, X_{m}\right)$ be a sequence of indeterminates; for every $a \in N^{m}$ we put $|a|=a_{1}+\cdots+a_{m}$ and $X^{a}=X_{1}^{a_{1}} \ldots X_{m}^{a_{m}}$, and we note that then $X^{a}$ is a monomial of (total) degree $|a|$. For all $a \in N^{m}$ and $b \in N^{m}$ we put $a+b=\left(a_{1}+b_{1}, \ldots, a_{m}+b_{m}\right) \in N^{m}$. Given any $f(X) \in R[X]$ we can uniquely write $f(X)=\sum_{a \in S} r_{a} X^{a}$ with $0 \neq r_{a} \in R$ for all $a \in S$, where $S$ (=

Received by the editors July 29, 1996 and, in revised form, November 8, 1996.

1991 Mathematics Subject Classification. Primary 12F10, 14H30, 20D06, 20 E22.

This work was partly supported by the Deutsche Forschungsgemeinschaft and the National Security Agency. 
the support of $f(X))$ is a finite subset of $N^{m}$. If $r_{a} \in R \backslash J$ for all $a \in S$, where $J$ is an ideal in $R$, then we shall say that $f(X)$ is a polynomial in $X$ over $(R, J)$. If also $a \not \leq a^{\prime}$ for all $a \neq a^{\prime}$ in $S$, then we shall say that $f(X)$ is a strict polynomial in $X$ over $(R, J)$. Note that every $f(X) \in R[X]$ is always a polynomial in $X$ over $(R, 0)$. For any $f(X) \in R[X]$, let $\bar{f}(X) \in(R / I)[X]$ be obtained by applying the canonical epimorphism $R \rightarrow R / I$ to the coefficients of $f(X)$. Given $j \in N$, we shall say that the sequence $x$ is $(j)$-separated if for every subset $V$ of $N^{m}$, upon letting $G$ to be the ideal in $R$ generated by $\left\{x^{b}: b \in V\right\}$, we have $\bigcap_{d=0}^{\infty}\left(I^{j} G+I^{d}\right)=I^{j} G$; if this is so for every finite subset $V$ of $N^{m}$ then we shall say that the sequence $x$ is finitely $(j)$-separated. We shall say that the sequence $x$ is monomially separated if it is (0)-separated. We shall say that the sequence $x$ is quasiseparated if it is finitely $(j)$-separated for some $j \in N$. Note that if $x$ is (resp: finitely) $(j)$-separated then it is (resp: finitely) $\left(j+j^{\prime}\right)$-separated for every $j^{\prime} \geq j$; this follows by noting that with $V$ and $G$ as above, upon letting $G^{\prime}$ be the ideal in $R$ generated by the (resp: finite) set $V^{\prime}=\left\{b+b^{\prime}: b \in V\right.$ and $b^{\prime} \in N^{m}$ with $\left.\left|b^{\prime}\right|=j^{\prime}\right\}$, we have $I^{j+j^{\prime}} G=I^{j} G^{\prime}$. It follows that

(2.0). $x$ is monomially separated $\Rightarrow x$ is quasiseparated $\Rightarrow$ given any $j^{*} \in N$ there exists $j^{*} \leq j^{* *} \in N$ such that $x$ is finitely $(j)$-separated whenever $j^{* *} \leq j \in N$.

Using the notion of quasiseparated, we shall now prove the following:

Existence Lemma (2.1). Assume that $x$ is quasiseparated. Then, given any $u \in$ $R$, there exists a strict polynomial $f(X)$ in $X$ over $(R, I)$ with $u=f(x)$; the polynomial $f(X)$ may be called a polyexpansion of $u$ relative to $(R, x)$, and the polynomial $\bar{f}(X)$ may be called a polyform of $u$ relative to $(R, x)$.

Actually we shall prove the following:

Strong Existence Lemma (2.2). Assume that $x$ is quasiseparated. Given any $W \subset N^{m}$, let $H$ be the ideal in $R$ generated by $\left\{x^{b}: b \in W\right\}$, and let $u \in H$. Then there exists a strict polynomial $f(X)$ in $X$ over $(R, I)$ with $u=f(x)$ such that for every $a$ in the support of $f(X)$ we have $b \leq a$ for some $b \in W$ (with $b$ depending on a).

Note that (2.1) follows by taking $W=\{(0, \ldots, 0)\}$ in (2.2), because then $H=R$. To prove (2.2), for $e=0,1,2, \ldots$ let $W_{e}=\left\{a \in N^{m}:|a|=e\right.$ and $b \leq a$ for some $b \in W\}$ and $H_{e}=$ the ideal in $R$ generated by $\left\{x^{a}: a \in W_{e}\right\}$, and for $d=0,1$, $2, \ldots$ let $H(d)=$ the ideal in $R$ generated by $\left\{x^{a}: a \in \bigcup_{d \leq e<\infty} W_{e}\right\}$. Since $u \in H$, we can find $S_{e} \subset W_{e}$ for $e=0,1,2, \ldots$ together with $t_{a} \in R \backslash I$ for all $a \in S_{e}$, such that upon letting $S(d)=\bigcup_{e=0}^{d-1} S_{e}$ and $u_{d}=\sum_{a \in S(d)} t_{a} x^{a}$ we have $u-u_{d} \in H(d)$ for $d=0,1,2, \ldots$ For $d=0$ this is obvious, because $S(0)=\emptyset$, and hence $u_{0}=0$ and $u-u_{0}=u \in H=H(0)$. Having done it thru a nonnegative integer $d$, and so having found $S_{0}, \ldots, S_{d-1}$, we have $u-u_{d} \in H(d)=H(d+1)+H_{d}$, and hence $u-u_{d}=h+\sum_{a \in W_{d}} v_{a} x^{a}$ with $h \in H(d+1)$ and $v_{a} \in R$ for all $a \in W_{d}$, and therefore, upon letting $S_{d}=\left\{a \in W_{d}: v_{a} \notin I\right\}$ and $t_{a}=v_{a}$ for all $a \in S_{d}$, we get $u-u_{d+1}=h+\sum_{a \in W_{d} \backslash S_{d}} v_{a} x^{a} \in H(d+1)$.

Let $K$ be any field. Let $G_{d}$ be the ideal in $K[X]$ generated by $\left\{X^{a}: a \in S(d)\right\}$. Then $G_{0} \subset G_{1} \subset G_{2} \subset \ldots$ is a sequence of ideals in the noetherian ring $K[X]$, and hence, by the chain condition, for some nonnegative integer $c$ we have $G_{d}=G_{c}$ for all integers $d \geq c$. It follows that for every $d \geq c$ and every $a^{*} \in S(d) \backslash S(c)$ we have $a<a^{*}$ for some $a \in S(c)$. In view of (2.0) we can take $0<j \in N$ such 
that $x$ is finitely $(j)$-separated. Let $u^{\prime}=\sum_{a \in S(c+j) \backslash S} t_{a} x^{a}$, where $S=\{a \in S(c)$ : $\widehat{a} \not \leq a$ whenever $a \neq \widehat{a} \in S(c)\}$. Then $S$ is a finite subset of $N^{m}$ such that for all $a \neq a^{\prime}$ in $S$ we have $a \not \leq a^{\prime}$, and such that for every $a^{*} \in S(c) \backslash S$ we have $a<a^{*}$ for some $a \in S$. It follows that for every $d \geq c+j$ and every $a^{*} \in S(d) \backslash S$ we have $a^{*}=a+\widetilde{a}$ for some $a \in S$ and $\widetilde{a} \in N^{m}$ with $|\widetilde{a}|=j$. Therefore, upon letting $G$ be the ideal in $R$ generated by $\left\{x^{a}: a \in S\right\}$, we have $u_{d}-u^{\prime} \in I^{j} G$ for every $d \geq c+j$. Since $u-u^{\prime}=\left(u-u_{d}\right)+\left(u_{d}-u^{\prime}\right)$ and $u-u_{d} \in H(d) \subset I^{d}$, we see that $u-u^{\prime} \in I^{j} G+I^{d}$ for every $d \geq c+j$. Now $\bigcap_{d=0}^{\infty}\left(I^{j} G+I^{d}\right)=I^{j} G$, and hence we get $u-u^{\prime} \in I^{j} G \subset I G$. Therefore we can write $u-u^{\prime}=\sum_{a \in S} t_{a}^{\prime} x^{a}$ with $t_{a}^{\prime} \in I$ for all $a \in S$. Since, for every $a^{*} \in S(c) \backslash S$ we have $a<a^{*}$ for some $a \in S$, we can also write $u^{\prime}=\sum_{a \in S} t_{a}^{*} x^{a}$, where $t_{a}^{*} \in R \backslash I$ for all $a \in S$. Let $f(X)=\sum_{a \in S} r_{a} X^{a}$, with $r_{a}=t_{a}^{*}+t_{a}^{\prime}$ for all $a \in S$. Then $r_{a} \in R \backslash I$ for all $a \in S$, and hence $f(X)$ is a strict polynomial in $X$ over $(R, I)$ with $u=f(x)$. Clearly the support of $f(X)$ is $S$; since $S \subset S(c) \subset \bigcup_{e=0}^{c-1} W_{e}$, it follows that for every $a \in S$ we have $b \leq a$ for some $b \in W$.

In a moment we shall prove the following:

Uniqueness Lemma (2.3). If $x$ is quasiseparated and quasiregular, then any $u \in$ $R$ has a unique polyform relative to $(R, x)$, which may hence be called the polyform of $u$ relative to $(R, x)$.

Recall that the sequence $x$ being quasiregular means $I \neq R$ and the graded ring $\operatorname{gr}_{I}(R)=\sum_{d=0}^{\infty}\left(I^{d} / I^{d+1}\right)$ is a polynomial ring over $R / I$ in the images of $x_{1}, \ldots, x_{m}$ in $I / I^{2}$. Also recall that the sequence $x$ being regular means $I \neq R$ and $x_{i}$ is a nonzerodivisor in $R / I_{i}$ for $1 \leq i \leq m$ where $I_{i}$ is the ideal in $R$ generated by $\left(x_{1}, \ldots, x_{i-1}\right)$. We shall say that the sequence $x$ is pseudoseparated if $\bigcap_{d=0}^{\infty}\left(I_{i}+I^{d}\right)=I_{i}$ for $1 \leq i \leq m$. Note that if $x$ is regular then it is quasiregular, and if $x$ is pseudoseparated and quasiregular then it is regular; cf. Theorem 27 on page 98 of [Mats]; clearly monomially separated $\Rightarrow$ pseudoseparated, and as we have already noted monomially separated $\Rightarrow$ quasiseparated, but we do not know whether there is an implication between pseudoseparated and quasiseparated; however, in (2.7) we shall show that quasiseparated + quasiregular $\Rightarrow$ monomially separated. Also note that if $R$ is a regular local ring and $x$ is part of a regular system of parameters, i.e., part of a minimal set of generators of the maximal ideal of $R$, then $x$ is monomially separated and quasiregular; cf. pages 301-304 of [ZarS]. The polynomial ring property of the graded ring is sharpened in the following:

Independence Lemma (2.4). If $x$ is quasiseparated and quasiregular, and $f(X)$ and $g(X)$ are strict polynomials in $X$ over $(R, I)$ such that $f(x)=g(x)$, then $\bar{f}(X)=\bar{g}(X)$.

Actually we shall prove the following:

Strong Independence Lemma (2.5). Assume that $x$ is quasiseparated and quasiregular. Let $f(X)$ and $g(X)$ be strict polynomials in $X$ over $(R, I)$, let $S$ and $T$ be their respective supports, let $J$ be the ideal in $R$ generated by $\left\{x^{a}: a \in S \cup T\right\}$, and let $d$ be a nonnegative integer such that $|a| \leq d$ for all $a \in S \cup T$. Assume that $f(x)-g(x) \in I J+I^{d+1}$. Then $\bar{f}(X)=\bar{g}(X)$.

Note that (2.4) follows from (2.5), whereas (2.3) follows from (2.1) and (2.4). Also note that the polynomial ring property of $\operatorname{gr}_{I}(R)$ corresponds to the special 
case of (2.5) when $|a|=d$ for all $a \in S \cup T$; observe that in this case we have $I J+I^{d+1}=I^{d+1}$.

To prove (2.5), suppose if possible that $\bar{f}(X) \neq \bar{g}(X)$. Then we can find $a^{\prime}$ in the support of $\bar{f}(X)-\bar{g}(X)$ together with a nonnegative integer $D \leq d$ such that $\left|a^{\prime}\right|=D \leq|a|$ for all $a$ in the support of $\bar{f}(X)-\bar{g}(X)$. Now $a^{\prime} \in S \cup T$, and upon letting $S(D)=\{a \in S:|a|<D\}$ and $T(D)=\{a \in T:|a|<D\}$ and $P(D)=\left\{a \in N^{m}:|a|=D\right\}$, we must have $S(D)=T(D)$, and we can write $f(x)-g(x)=\sum_{a \in S(D) \cup P(D)} t_{a} x^{a}$ with $t_{a} \in I$ for all $a \in S(D)$, and $t_{a} \in R$ for all $a \in P(D)$, and $t_{a^{\prime}} \notin I$. Since by assumption $f(x)-g(x) \in I J+I^{d+1}$, we can also write $f(x)-g(x)=\sum_{a \in S(D) \cup P(D)} t_{a}^{\prime} x^{a}$ with $t_{a}^{\prime} \in I$ for all $a \in S(D) \cup P(D)$. Let $r_{a}=t_{a}-t_{a}^{\prime}$ for all $a \in S \cup T$. Then $\sum_{a \in S(D) \cup P(D)} r_{a} x^{a}=0$ with $r_{a} \in I$ for all $a \in S(D)$, and $r_{a} \in R$ for all $a \in P(D)$, and $r_{a^{\prime}} \notin I$. Let $W=\{b \in$ $N^{m}: b=\left(a_{1}, \ldots, a_{i-1}, 1+a_{i}, a_{i+1}, \ldots, a_{m}\right)$ for some $a \in S(D)$ and some $i \in$ $\{1, \ldots, m\}$ (with $a$ and $i$ depending on $b$ ) $\}$, and let $H$ be the ideal in $R$ generated by $\left\{x^{b}: b \in W\right\}$. Then $\sum_{a \in S(D)} r_{a} x^{a} \in H$, and hence by (2.2) we get $\sum_{a \in S(D)} r_{a} x^{a}=\sum_{j \in S^{\prime}} s_{j} x^{j}$ with $s_{j} \in R \backslash I$ for all $j \in S^{\prime}$, where $S^{\prime}$ is a finite subset of $N^{m}$ such that: (') for every $j \in S^{\prime}$ we have $a<j$ for some $a \in S(D)$. In view of the facts that $S(D)=T(D) \subset S \cap T$ and $a^{\prime} \in S \cup T$, where $S$ and $T$ are the respective supports of $f(X)$ and $g(X)$ which are strict polynomials in $X$ over $(R, I)$, property $\left({ }^{\prime}\right)$ tells us that $a^{\prime} \notin S^{\prime}$.

Note that now $\sum_{a \in P(D)} r_{a} x^{a}=-\sum_{j \in S^{\prime}} s_{j} x^{j}$ with $r_{a} \in R$ for all $a \in P(D)$, and $r_{a^{\prime}} \notin I$ for some $a^{\prime} \in P(D)$, and $s_{j} \in R$ for all $j \in S^{\prime}$. If $|j|<D$ for some $j \in S^{\prime}$, then we could take a nonnegative integer $E<D$ such that $|j| \geq E$ for all $j \in S^{\prime}$, and such that upon letting $S^{*}=\left\{j \in S^{\prime}:|j|=E\right\}$ we have $S^{*} \neq \emptyset$, and then we would get $\sum_{j \in S^{*}} s_{j} x^{j} \in I^{E+1}$, which would contradict the polynomial ring property of $\operatorname{gr}_{I}(R)$. If $|j| \geq D$ for all $j \in S^{\prime}$, then upon letting $P^{\prime}(D)=\left\{j \in S^{\prime}:|j|=D\right\}$ we would get $\sum_{a \in P(D)} r_{a} x^{a}=-s^{*}-\sum_{j \in P^{\prime}(D)} s_{j} x^{j}$ with $s^{*}=\sum_{j \in S^{\prime} \backslash P^{\prime}(D)} s_{j} x^{j} \in$ $I^{D+1}$; since $a^{\prime} \in P(D) \backslash P^{\prime}(D)$, this would again contradict the polynomial ring property of $\operatorname{gr}_{I}(R)$. Therefore we must have $\bar{f}(X)=\bar{g}(X)$.

As an immediate consequence of Lemmas (2.2) and (2.3) we have the following:

Corollary (2.6). Assume that $x$ is quasiseparated and quasiregular. Given any $W^{(l)} \subset N^{m}$, with $l$ varying in any indexing set $L$, let $H^{(l)}$ be the ideal in $R$ generated by $\left\{x^{b}: b \in W^{(l)}\right\}$, let $\widehat{W}^{(l)}=\left\{a \in N^{m}: b \leq a\right.$ for some $\left.b \in W^{(l)}\right\}$, let $\widehat{W}=$ $\bigcap_{l \in L} \widehat{W}^{(l)}$, let $\widehat{H}$ be the ideal in $R$ generated by $\left\{x^{a}: a \in \widehat{W}\right\}$, and let $H$ be the set of all $u \in R$ such that the support of the polyform of $u$ relative to $(R, x)$ is contained in $\widehat{W}$. Then $H=\widehat{H}=\bigcap_{l \in L} H^{(l)}$.

Given any ideal $G$ in $R$ generated by $\left\{x^{b}: b \in V\right\}$, where $V$ is any subset of $N^{m}$, upon letting $L=N$ and $W^{(d)}=V \cup\left\{b \in N^{m}:|b|=d\right\}$ for all $d \in N$, by (2.6) we see that if $x$ is quasiseparated and quasiregular then $\bigcap_{d=0}^{\infty}\left(G+I^{d}\right)=G$; consequently, in view of Theorem 27 on page 98 of [Mats] we get the following:

Corollary (2.7). Assume that $x$ is quasiseparated and quasiregular. Then $x$ is monomially separated and regular.

\section{Section 3: Concavity}

Again let $I$ be the ideal generated by a finite sequence $x=\left(x_{1}, \ldots, x_{m}\right)$ in a ring $R$. Recall that the support of a strict polynomial $f(X)$ in $X$ over $(R, I)$ is a subset 
$S$ of $N^{m}$ such that for all $a \neq a^{\prime}$ in $S$ we have $a \not \leq a^{\prime}$. Let us call a subset of $N^{m}$ (or any poset) concave if it has this property.

By induction on $m$, we can see that a concave subset of $N^{m}$ is necessarily finite. For $m \leq 1$, this is obvious because then a concave subset has at most 1 element. So let $m>1$ and assume it for $m-1$. Let if possible $S$ be an infinite concave subset of $N^{m}$. Fix $a \in S$. For $1 \leq i \leq m$ and any nonnegative integer $j$ let $S_{i j}=\left\{b \in S: b_{i}=j\right\}$. Also let $S^{\prime}=\bigcup_{1 \leq i \leq m} \bigcup_{0 \leq j \leq a_{i}} S_{i j}$. Now a subset of a concave set is concave, and the projection in $N^{m-1}$ of a concave set in $N^{m}$ with constant $i$-th coordinate is concave. Therefore, by the induction hypothesis, each $S_{i j}$ is finite, and hence so is $S^{\prime}$. Therefore there exists $a^{\prime} \in S \backslash S^{\prime}$. This is a contradiction, because then $a<a^{\prime}$.

For every concave $S \subset N^{m}$, let $R[X]_{(I, S)} \subset R[X]$ be the set of all polynomials in $X$ over $(R, I)$ with support $S$. Then $R[X]_{(I, S)}$ can be identified with $(R \backslash I)^{S}=$ the set of all maps of $S$ into $R \backslash I$. Likewise, upon letting $\bar{R}=R / I$, the set $\bar{R}[X]_{(0, S)}$ can be identified with the set $(\bar{R} \backslash 0)^{S}$. Let $R[X]_{(I)} \subset R[X]$ be the set of all strict polynomials in $X$ over $(R, I)$, and let $\left(N^{m}\right)^{*}$ be the set of all concave subsets of $N^{m}$. Then we have the disjoint partition representations $R[X]_{(I)}=\amalg_{S \in\left(N^{m}\right)^{*}} R[X]_{(I, S)}$ and $\bar{R}[X]_{(0)}=\amalg_{S \in\left(N^{m}\right) *} \bar{R}[X]_{(0, S)}$. The canonical epimorphism $\phi: R \rightarrow \bar{R}$ induces an epimorphism $\Phi: R[X] \rightarrow \bar{R}[X]$. Note that $\Phi\left(R[X]_{(I)}\right)=\bar{R}[X]_{(0)}$, and for every $S \in\left(N^{m}\right)^{*}$ we have $\Phi\left(R[X]_{(I, S)}\right)=\bar{R}[X]_{(0, S)}$. By sending $X$ to $x$ we get an $R$ homomorphism $\sigma: R[X] \rightarrow R$. Lemma (2.1) says that if $x$ is monomially separated, then $\sigma\left(R[X]_{(I)}\right)=R$. Lemma (2.3) says that if $x$ is monomially separated and quasiregular, then by sending every $u \in R$ to its polyform relative to $(R, x)$ we get a map $\rho: R \rightarrow \bar{R}[X]$ such that for every $F \in R[X]_{(I)}$ we have $\rho(\sigma((F))=\Phi(F)$, and hence in particular $\rho(R)=\bar{R}[X]_{(0)}$; note that in this case, upon letting $S$ be the support of the polyform of $u \in R$ relative to $(R, x)$, we have that: (i) $S$ is also the support of every polyexpansion of $u$ relative to $(R, x)$; (ii) $u=0 \Leftrightarrow S=\emptyset$; and (iii) $u \neq 0 \Rightarrow u \in I^{d} \backslash I^{d+1}$, where $d=\min \{|a|: a \in S\}$, and the sum of the terms of degree $d$ in the polyform of $u$ relative to $(R, x)$ is the initial form of $u$ relative to $(R, x)$, i.e., the image of $u$ in $I^{d} / I^{d+1}$ as a homogeneous polynomial over $R / I$ in the images of $x_{1}, \ldots, x_{m}$ in $I / I^{2}$ (the initial form of 0 is taken to be 0 ).

At any rate, the combinatorics of concave sets should be quite significant for desingularization.

\section{SeCtion 4: Order AND COEFFICIENT SET}

The maximal ideal of a (noetherian) local ring $R$ is denoted by $M(R)$. For any $0 \neq u \in R$ we define $\operatorname{ord}_{R} u$ to be the unique nonnegative integer such that $u \in M(R)^{d} \backslash M(R)^{d+1}$; also we put $\operatorname{ord}_{R} 0=\infty$. For any $H \subset R$ we define $\operatorname{ord}_{R} H=\min \left\{\operatorname{ord}_{R} u: u \in H R\right\}$, with the convention $\operatorname{ord}_{R} H=\infty \Leftrightarrow H R=0$. Given any $L \in R$ or $L \subset M(R)$, upon letting $\psi: R \rightarrow R / L R$ be the canonical epimorphism, for any $H \in R$ or $H \subset R$ we define $\operatorname{ord}_{R / L} H=\operatorname{ord}_{\psi(R)} \psi(H)$. By a coefficient set for $R$ we mean a subset of $R$ which contains 0 and 1 and which is mapped bijectively onto $R / M(R)$ by the canonical epimorphism $R \rightarrow R / M(R)$. Note that if $R$ is complete and has the same characteristic as $R / M(R)$, then, by Cohen's theorem, $R$ has a coefficient field, i.e., a coefficient set which is a subfield of $R$. But obviously we can always find a coefficient set for any local ring $R$. 


\section{Section 5: Magnitude and Shreedharacharya EXPansion}

Let $R$ be a 2 dimensional regular local ring, and let $J=u R$ be a nonzero principal ideal in $R$ with $0 \neq u \in R$. Let $\phi: R \rightarrow \bar{R}=R / M(R)$ be the canonical epimorphism, let $k$ be a coefficient set for $R$, and let $(x, y)$ be a basis of $M(R)$. Let $d=\operatorname{ord}_{R} J$ and $D=\operatorname{ord}_{R / x} J$. Note that then $d$ is a nonnegative integer with $d \leq D$, and $D$ is either a nonnegative integer or $\infty$.

Let $f(X, Y)$ be a polyexpansion of $u$ relative to $(R,(x, y))$. Then $f(X, Y)=$ $\sum_{(i, j) \in S} r_{i j} X^{i} Y^{j}$ with $r_{i j} \in R \backslash M(R)$ for all $(i, j) \in S$, where the nonempty finite (concave) set $S \subset N \times N$ is the support of $f(X, Y)$. Let $\bar{f}(X, Y)$ be the polyform of $u$ relative to $(R,(x, y))$. Note that then the support of $\bar{f}(X, Y)$ is also $S$, and we have $\bar{f}(X, Y)=\sum_{(i, j) \in S} \phi\left(r_{i j}\right) X^{i} Y^{j}$. Also note that $D=\infty \Leftrightarrow i>0$ for all $(i, j) \in S$.

If $D \neq \infty$, then we can uniquely write

$$
f(X, Y)=u_{0} Y^{D}+\sum_{j \in T} u_{j} X^{s(j)} Y^{D-j}
$$

with $u_{0} \in R \backslash M(R)$, and $0<s(j) \in N$ and $u_{j} \in R \backslash M(R)$ for all $j \in T$, where $T \subset\{1,2, \ldots, D\}$ is such that $S=\{(0, D)\} \cup\{(s(j), D-j): j \in T\}$, and upon letting $U_{0}=\phi\left(u_{0}\right)$ and $U_{j}=\phi\left(u_{j}\right)$ for all $j \in T$ we have

$$
\bar{f}(X, Y)=U_{0} Y^{D}+\sum_{j \in T} U_{j} X^{s(j)} Y^{D-j}
$$

with $0 \neq U_{0} \in \bar{R}$ and $0 \neq U_{j} \in \bar{R}$ for all $j \in T$. Note that

$(\bullet)$. If $J=u^{\bullet} R$ with $0 \neq u \bullet \in R$ and $\bar{f}^{\bullet}(X, Y)$ is the polyform of $u^{\bullet}$ relative to $(R,(x, y))$, then obviously $0 \neq \bar{f}^{\bullet}(X, Y) / \bar{f}(X, Y) \in \bar{R}$ and hence, in particular, the support of $\bar{f}^{\bullet}(X, Y)$ coincides with the support $S$ of $\bar{f}(X, Y)$.

Let

$$
e= \begin{cases}0 & \text { if } D=\infty \\ \infty & \text { if } D \neq \infty \text { and } T=\emptyset \\ \min \{s(j) / j: j \in T\} & \text { if } D \neq \infty \text { and } T \neq \emptyset\end{cases}
$$

and note that then $e$ is a nonnegative rational or $\infty$, and we have $e=0 \Leftrightarrow D=\infty$, and also $e=\infty \Leftrightarrow D \neq \infty$ and $T=\emptyset$.

If $0<e<\infty$, then upon letting $T^{\sharp}=\{j \in T: s(j) / j=e\}$ and

$$
f^{\sharp}(X, Y)=u_{0} Y^{D}+\sum_{j \in T^{\sharp}} u_{j} X^{s(j)} Y^{D-j} \text { and } f^{b}(X, Y)=\sum_{j \in T \backslash T^{\sharp}} u_{j} X^{s(j)} Y^{D-j}
$$

we see that

$$
f(X, Y)=f^{\sharp}(X, Y)+f^{b}(X, Y) \text { with } f^{\sharp}(X, Y) \neq 0,
$$

and upon letting

$$
F^{\sharp}(X, Y)=U_{0} Y^{D}+\sum_{j \in T^{\sharp}} U_{j} X^{s(j)} Y^{D-j} \text { and } F^{b}(X, Y)=\sum_{j \in T \backslash T^{\sharp}} U_{j} X^{s(j)} Y^{D-j}
$$

we see that

$$
\bar{f}(X, Y)=F^{\sharp}(X, Y)+F^{b}(X, Y) \text { with } F^{\sharp}(X, Y) \neq 0
$$


and, giving weights $(1, e)$ to $(X, Y)$, every term in $F^{\sharp}(X, Y)$ has weight $D e$ whereas every term in $F^{b}(X, Y)$ has weight $>D e$ and so, by definition, $F^{\sharp}(X, Y)$ is the weighted initial form of $\bar{f}(X, Y)$.

Note that if $0<e<\infty$ and we give weights $(1, \pi)$ to $(X, Y)$ with nonnegative rational $\pi<e$, then the weighted initial form of $\bar{f}(X, Y)$ equals $U_{0} Y^{D}$. Likewise, if $0<e<\infty$ and we give weights $(1, \pi)$ to $(X, Y)$ with rational $\pi>e$, then the weighted initial form of $\bar{f}(X, Y)$ does not contain $U_{0} Y^{D}$. Finally, as we have already noted, if $0<e<\infty$ and we give weights $(1, e)$ to $(X, Y)$, then the weighted initial form of $\bar{f}(X, Y)$ contains $U_{0} Y^{D}$ but is not equal to it. This gives an alternative characterization of $e$.

If $0<e \in N$, then obviously either $F^{\sharp}(X, Y) \neq U_{0}\left(Y+\widetilde{C} X^{e}\right)^{D}$ for all $0 \neq \widetilde{C} \in \bar{R}$, or $F^{\sharp}(X, Y)=U_{0}\left(Y+\widetilde{C} X^{e}\right)^{D}$ for a unique $0 \neq \widetilde{C} \in \bar{R}$; cf. Lemma (5.3) on page 14 of $[\mathrm{AbhD}]$. Let

$$
C= \begin{cases}0 & \text { if either } e=0 \text { or } 0<e \notin N, \\ 0 & \text { if } 0<e \in N \text { and } F^{\sharp}(X, Y) \neq U_{0}\left(Y+\widetilde{C} X^{e}\right)^{D} \text { for all } 0 \neq \widetilde{C} \in \bar{R}, \\ \widetilde{C} & \text { if } 0<e \in N \text { and } F^{\sharp}(X, Y)=U_{0}\left(Y+\widetilde{C} X^{e}\right)^{D} \text { with } 0 \neq \widetilde{C} \in \bar{R},\end{cases}
$$

and let $c$ be the unique element in $k$ such that

$$
\phi(c)=C \text {. }
$$

We define the magnitude $\operatorname{mag}(R, J, x, y)$ by putting

$$
\operatorname{mag}(R, J, x, y)=e,
$$

and the Shreedharacharya coefficients $\operatorname{sic}(R, J, x, y)$ and $\operatorname{sic}(R, J, x, y, k)$ by putting

$$
\operatorname{sic}(R, J, x, y)=C \quad \text { and } \quad \operatorname{sic}(R, J, x, y, k)=c .
$$

Shreedharacharya was the fifth century Indian mathematician whose completing the square method of solving quadratic equations is given in verse 116 of Bhaskaracharya's Beejaganita of 1150 A.D. [Bhas]. Our definition of $C$ is inspired by the analogous completing the $D$-th power procedure. However, our $C$ does NOT involve any division by $D$, because we take a nonzero $C$ only if we can complete the $D$-th power without doing any work!

By $(\bullet)$ we see that the quantities $e$ and $C$ depend only on $J$ and the basis $(x, y)$ but not on the particular generator $u$ of $J$. For the quantities $d, D, e, C$, here are some:

Obvious Properties (5.1). (5.1.1) $d \in N$ and $d \leq D \in N \cup\{\infty\}$. (5.1.2) $e \in\{$ nonnegative rationals $\} \cup\{\infty\}$ and $C \in \bar{R}$. (5.1.3) $e=0 \Leftrightarrow D=\infty$. (5.1.4) $e=\infty \Leftrightarrow D \neq \infty$ and $J=y^{D} R$. (5.1.6) $d=0 \Rightarrow e=\infty$. (5.1.7) $0<e<\infty \Rightarrow$ $1 \leq D<\infty$ and $0<D$ ! e $\in N$. (5.1.8) $e \geq 1 \Leftrightarrow D=d$. (5.1.9) $e>1 \Leftrightarrow J \subset$ $y^{d} R+M(R)^{d+1}$. (5.1.10) $C \neq 0 \Leftrightarrow 0<e \in N$ and $F^{\sharp}(X, Y)=U_{0}\left(Y+\widetilde{C} X^{e}\right)^{D}$ with $0 \neq \widetilde{C} \in \bar{R}$. (5.1.11) $\operatorname{mag}\left(R, J, x^{*}, y^{*}\right)>1$ for some basis $\left(x^{*}, y^{*}\right)$ of $M(R) \Leftrightarrow J \subset$ $y^{* d}+M(R)^{d+1}$ for some $y^{*} \in M(R) \backslash M(R)^{2}$. (5.1.12) $e=1$ and $C \neq 0 \Leftrightarrow e=1$ and $J \subset y^{* d}+M(R)^{d+1}$ for some $y^{*} \in M(R) \backslash M(R)^{2}$.

The only items in this which may not be obvious are (5.1.11) and (5.1.12). The former follows from (5.1.9) by noting that, given any $y^{*} \in M(R) \backslash M(R)^{2}$, we can always take $x^{*} \in M(R) \backslash M(R)^{2}$ with $\left(x^{*}, y^{*}\right) R=M(R)$. Likewise (5.1.12) follows from (5.1.10) by taking into account (5.1.6) and (5.1.8). 
In the next three propositions we shall investigate change of magnitude with change of basis; cf. Proposition (8.1) and Lemmas (8.4)-(8.7) on pages 26-33 of [AbhD].

Proposition (5.2). Let $\left(x^{*}, y^{*}\right)$ be a basis of $M(R)$ such that $x=g x^{*}+\widetilde{g} y^{*}$ and $y=h y^{*}+\widetilde{h} x^{* E}$ with $g \in R \backslash M(R), \widetilde{g} \in R, h \in R \backslash M(R), \widetilde{h} \in R$, and $0<E \in N$. Let $G=\phi(g), \widetilde{G}=\phi(\widetilde{g}), H=\phi(h), \widetilde{H}=\phi(\widetilde{h}), D^{*}=\operatorname{ord}_{R / x^{*}} J$, $e^{*}=\operatorname{mag}\left(R, J, x^{*}, y^{*}\right)$, and $C^{*}=\operatorname{sic}\left(R, J, x^{*}, y^{*}\right)$. Assume that $e \geq 1$. Then $D=d$, and we have the following. (5.2.1) If $E>e \notin N$, then $D^{*}=D$ and $e^{*}=e$ and $C^{*}=C=0$. (5.2.2) If $E>e \in N$, then $D^{*}=D$ and $e^{*}=e$ and $C^{*}=C G^{e} / H$. (5.2.3) If $E<e$ and $D \neq 0 \neq \widetilde{H}$, then $D^{*}=D$ and $e^{*}=E$ and $C^{*}=\widetilde{H} / H$. (5.2.4) If $E=e>1$ and $C=0$, then $D^{*}=D$ and $e^{*}=e$ and $C^{*}=0$. (5.2.5) If $E=e>1$ and $C \neq 0 \neq \widetilde{H}+C G^{e}$, then $D^{*}=D$ and $e^{*}=e$ and $C^{*}=\left(\widetilde{H}+C G^{e}\right) / H$. (5.2.6) If $E=e>1$ and $C \neq 0=\widetilde{H}+C G^{e}$, then $D^{*}=D$ and $e^{*}>e$. (5.2.7) If $E=e=1$ and $C \neq 0=\widetilde{G}=0=\widetilde{H}+C G$, then $D^{*}=D$ and $e^{*}>1$.

To see this, upon letting $f^{\natural}(X, Y)=f\left(g X+\widetilde{g} Y, h Y+\widetilde{h} X^{E}\right)$ we have $u=$ $f^{\natural}\left(x^{*}, y^{*}\right)$. Let $f^{*}(X, Y)$ be a polyexpansion of $u$ relative to $\left(R,\left(x^{*}, y^{*}\right)\right)$, and let $\bar{f}^{*}(X, Y)$ be obtained by applying $\phi$ to the coefficients of $f^{*}(X, Y)$, i.e., let $\bar{f}^{*}(X, Y)$ be the polyform of $u$ relative to $\left(R,\left(x^{*}, y^{*}\right)\right)$. Since by assumption $e \geq 1$, we must have $D=d$. For any nonnegative rational number $\pi$ let $\sum^{(\pi)}$ denote finite summation over $(i, j)$ in $N \times N$ with $i+j \pi>D \pi$, where the specific finite indexing set is allowed to vary from formula to formula. If $E>e$ then $f^{\natural}(X, Y)=$ $f^{\sharp}(g X, h Y)+\sum^{(e)} t_{i j} X^{i} Y^{j}$ with $t_{i j} \in R$, and hence by (2.6) we see that $\bar{f}^{*}(X, Y)=$ $F^{\sharp}(G X, H Y)+\sum^{(e)} W_{i j} X^{i} Y^{j}$ with $0 \neq W_{i j} \in \bar{R}$, and therefore $D^{*}=D$ and $e^{*}=e$, and if $e \notin N$ then $C^{*}=C=0$ whereas if $e \in N$ then $C^{*}=C G^{e} / H$. If $E<$ $e$ and $D \neq 0 \neq \widetilde{H}$, then $f^{\natural}(X, Y)=u_{0}\left(h Y+\widetilde{h} X^{E}\right)^{D}+\sum^{(E)} t_{i j} X^{i} Y^{j}$ with $t_{i j} \in R$, and hence by (2.6) we see that $\bar{f}^{*}(X, Y)=U_{0}\left(H Y+\widetilde{H} X^{E}\right)^{D}+\sum^{(E)} W_{i j} X^{i} Y^{j}$ with $0 \neq W_{i j} \in \bar{R}$, and therefore $D^{*}=D$ and $e^{*}=E$ and $C^{*}=\widetilde{H} / H$. If $E=e$ and either $e-1>0$ or $e-1=0=\widetilde{G}$, then $f^{\natural}\left(x^{*}, y^{*}\right)=f^{\text {घh }}\left(x^{*}, y^{*}\right)$, where $f^{\text {घh }}(X, Y)=$ $f^{\sharp}\left(g X, h Y+\widetilde{h} X^{e}\right)+\sum^{(e)} t_{i j} X^{i} Y^{j}$ with $t_{i j} \in R$, and hence by (2.6) we see that $\bar{f}^{*}(X, Y)=F^{\sharp}\left(G X, H Y+\widetilde{H} X^{e}\right)+\sum^{(e)} W_{i j} X^{i} Y^{j}$ with $0 \neq W_{i j} \in \bar{R}$, and therefore $D^{*}=D$, and if $C=0$, then $e^{*}=e$ and $C^{*}=0$, whereas if $C \neq 0 \neq \widetilde{H}+C G^{e}$, then $e^{*}=e$ and $C^{*}=\left(\widetilde{H}+C G^{e}\right) / H$, and likewise if $C \neq 0=\widetilde{H}+C G^{e}$ then $e^{*}>e$.

Proposition (5.3). If $e \geq 1$ and $C=0$, then for any basis $\left(x^{*}, y^{*}\right)$ of $M(R)$, upon letting $e^{*}=\operatorname{mag}\left(R, J, x^{*}, y^{*}\right)$, we have $e^{*} \leq e$, and if $e^{*} \geq 1$, then, upon letting $C^{*}=\operatorname{sic}\left(R, J, x^{*}, y^{*}\right)$, we have $e^{*}=e \Leftrightarrow C^{*}=0$.

To see this, we may assume that $e \geq 1 \leq e^{*}$ and $C=0$. The case of $d=0$ follows from (5.1.6) and (5.1.10). So we may also assume that $d \geq 1$. Now the case when either $e=1$ or $e^{*}=1$ follows from (5.1.12). So we may also assume that $e>1<e^{*}$. Now upon letting $s=\operatorname{ord}_{R / y^{*}} y$, in view of (5.1.9), we see that $s>1$. If $e=\infty=s$ then we are done by (5.1.4) and (5.1.10). So also assume that either $e \neq \infty$ or $s \neq \infty$. If $s \neq \infty$ then let $E=s$, and if $s=\infty$ then let $E$ be an integer $>e$. Now $y=h y^{*}+\widetilde{h} x^{* E}$ with $h$ and $\widetilde{h}$ in $R$ such that if $s \neq \infty$ then $\widetilde{h} \notin M(R)$. Since $E>1$, we must have $h \notin M(R)$. Since $E>1$, we must also have $x=g x^{*}+\widetilde{g} y^{*}$ with $g \in R \backslash M(R)$ and $\widetilde{g} \in R$. Now we are done by (5.2.1)-(5.2.4). 
Proposition (5.4). If $c \neq 0$ then $D$ and $e$ are positive integers, and upon letting $y^{*}=y+c x^{e}$ we have $M(R)=\left(x, y^{*}\right) R$ and $\operatorname{mag}\left(R, J, x, y^{*}\right)>e$.

This is simply a special case of (5.2.6) and (5.2.7).

Now if $\left(c_{0}, e_{0}\right)=(c, e)$ and $c_{0} \neq 0$, then, upon letting $y_{1}=y+c_{0} x^{e_{0}}$ and $e_{1}=\operatorname{mag}\left(R, J, x, y_{1}\right)$, by (5.4) we have $e_{1}>e_{0}$. If also $c_{1} \neq 0$, where $c_{1}=$ $\operatorname{sic}\left(R, J, x, y_{1}, k\right)$, then, upon letting $y_{2}=y+c_{0} x^{e_{0}}+c_{1} x^{e_{1}}$ and $e_{2}=\operatorname{mag}\left(R, J, x, y_{2}\right)$, by (5.4) we have $e_{2}>e_{1}$. And so on. Now either this process stops after a finite number of steps, or it continues for an infinite number of steps. In the finite case we get a nonnegative integer $\omega$ together with $c_{i} \in k$ and $e_{i} \in\{$ nonnegative rationals $\} \cup$ $\{\infty\}$ for $0 \leq i \leq \omega$ such that $\left(c_{0}, e_{0}\right)=(c, e)$ and $c_{i} \neq 0<e_{i} \in N$ and $e_{i+1}>e_{i}$ for $0 \leq i<\omega$, and such that upon letting $y_{i}=y+\sum_{0 \leq j<i} c_{j} x^{e_{j}}$ for $0<i \leq \omega$ we have $\left(x, y_{i}\right) R=M(R)$ and $e_{i}=\operatorname{mag}\left(R, J, x, y_{i}\right)$ and $c_{i}=\operatorname{sic}\left(R, J, x, y_{i}, k\right)$ for $0<i \leq \omega$, and such that $c_{\omega}=0$. In the infinite case, by letting $\omega=\infty$, we have $c_{i} \in k$ and $e_{i} \in\{$ nonnegative rationals $\} \cup\{\infty\}$ for $0 \leq i \leq \omega$ such that $\left(c_{0}, e_{0}\right)=(c, e)$ and $c_{i} \neq 0<e_{i} \in N$ and $e_{i+1}>e_{i}$ for $0 \leq i<\omega$, and such that upon letting $y_{i}=y+\sum_{0 \leq j<i} c_{j} x^{e_{j}}$ for $0<i \leq \omega$ we have $\left(x, y_{i}\right) R=M(R)$ and $e_{i}=\operatorname{mag}\left(R, J, x, y_{i}\right)$ and $c_{i}=\operatorname{sic}\left(R, J, x, y_{i}, k\right)$ for $0<i<\omega$, and such that $c_{\omega}=0$ and $e_{\omega}=\infty$; note that now $\left(x, y_{\omega}\right) \widehat{R}=M(\widehat{R})$, where $\widehat{R}$ is the completion of $R$, and we have $J \widehat{R} \subset \bigcap_{0 \leq i<\infty}\left(y_{i}^{D} \widehat{R}+M(\widehat{R})^{i}\right) \subset \bigcap_{0 \leq i<\infty}\left(y_{\omega}^{D} \widehat{R}+M(\widehat{R})^{i}\right)=y_{\omega}^{D} \widehat{R}$, and hence $J \widehat{R}=y_{\omega}^{D} \widehat{R}=y_{\omega}^{d} \widehat{R}$, and therefore $e_{\omega}=\operatorname{mag}\left(\widehat{R}, J \widehat{R}, x, y_{\omega}\right)$ and $c_{\omega}=$ $\operatorname{sic}\left(\widehat{R}, J \widehat{R}, x, y_{\omega}, k\right)$.

Thus we have proved the following theorem; cf. Theorem (9.8) on page 36 of [AbhD].

Shreedharacharya Expansion Theorem (5.5). There exists a unique $\omega \in N \cup$ $\{\infty\}$ together with unique $c_{i} \in k$ and $e_{i} \in\{$ nonnegative rationals $\} \cup\{\infty\}$ for $0 \leq i \leq$ $\omega$ such that $\left(c_{0}, e_{0}\right)=(c, e)$ and $c_{i} \neq 0<e_{i} \in N$ and $e_{i+1}>e_{i}$ for $0 \leq i<\omega$, and such that upon letting $y_{i}=y+\sum_{0 \leq j<i} c_{j} x^{e_{j}}$ for $0<i \leq \omega$ we have $\left(x, y_{i}\right) R=M(R)$ and $e_{i}=\operatorname{mag}\left(R, J, x, y_{i}\right)$ and $c_{i}=\operatorname{sic}\left(R, J, x, y_{i}, k\right)$ for $0<i<\omega$, and such that if $\omega \neq \infty$ then we have $\left(x, y_{\omega}\right) R=M(R)$ and $e_{\omega}=\operatorname{mag}\left(R, J, x, y_{\omega}\right)$ and $c_{\omega}=$ $\operatorname{sic}\left(R, J, x, y_{\omega}, k\right)=0$, whereas if $\omega=\infty$ then upon letting $\widehat{R}$ to be the completion of $R$ we have $\left(x, y_{\omega}\right) \widehat{R}=M(\widehat{R})$ and $J \widehat{R}=y_{\omega}^{d} \widehat{R}$ and $e_{\omega}=\operatorname{mag}\left(\widehat{R}, J \widehat{R}, x, y_{\omega}\right)=\infty$ and $c_{\omega}=\operatorname{sic}\left(\widehat{R}, J \widehat{R}, x, y_{\omega}, k\right)=0$.

In effect, (5.5) amounts to solving $f(X, Y)=0$ to express $Y$ as a function of $X$, as far as we can, without getting into fractional exponents. At any rate, $Y=$ $-\sum_{0 \leq j<\omega} c_{j} X^{j}$ is the best possible such expression; note that this is a complete solution of $f(X, Y)=0 \Leftrightarrow e_{\omega}=\infty$. In case of characteristic zero, our solution gives the initial part of the Newton expansion before fractional exponents come into play. One advantage of the above Shreedharacharya method is that it works in any characteristic, including mixed characteristic.

Recall that a ring $A$ is said to be pseudogeometric if $A$ is noetherian and, for any prime ideal $P$ in $A$, the integral closure of $A / P$ in any finite algebraic field extension of the quotient of $A / P$ is a finite $(A / P)$-module. Note that every field is pseudogeometric, and every Dedekind domain of characteristic zero is pseudogeometric, and every homomorphic image of a pseudogeometric ring is pseudogeometric. Also note that every complete local ring is pseudogeometric, every finitely generated ring extension of a pseudogeometric ring is pseudogeometric, and 
the localization of a pseudogeometric ring with respect to any multiplicative set in it is pseudogeometric; cf. (17.9), (32.1) and (36.1) of [Naga]. In connection with the $w=\infty$ case of (5.5) we note the following additional fact about pseudogeometric local rings; cf. Proposition (9.10) on page 38 of [AbhD].

Proposition (5.6). If $R$ is pseudogeometric and $J \widehat{R}=y^{* d} \widehat{R}$ for some $y^{*} \in M(\widehat{R}) \backslash$ $M(\widehat{R})^{2}$ where $\widehat{R}$ is the completion of $R$, then $J=y^{* d} R$ for some $y^{*} \in M(R) \backslash$ $M(R)^{2}$.

Let

$$
B(R, J)=\left\{\left(x^{*}, y^{*}\right):\left(x^{*}, y^{*}\right) R=M(R) \text { and } \operatorname{ord}_{R / x^{*}}=d\right\}
$$

and

$$
\widehat{e}= \begin{cases}\max \left\{\operatorname{mag}\left(R, J, x^{*}, y^{*}\right):\left(x^{*}, y^{*}\right) \in B(R, J)\right\} & \text { if } B(R, J) \neq \emptyset, \\ 1 & \text { if } B(R, J)=\emptyset,\end{cases}
$$

where max denotes least upper bound. We define the magnitude mag $[R](J)$ by putting

$$
\operatorname{mag}[R](J)=\widehat{e} .
$$

In view of (5.1), by (5.3)-(5.6) we get the following proposition, giving alternative characterizations of $\widehat{e}$.

Proposition (5.7). (5.7.1) $\widehat{e} \in\{$ rationals $\geq 1\} \cup\{\infty\}$. (5.7.2) $\widehat{e}>1 \Leftrightarrow J \subset y^{* d}+$ $M(R)^{d+1}$ for some $y^{*} \in M(R) \backslash M(R)^{2}$. (5.7.3) $\widehat{e} \neq \infty \Rightarrow d \geq 1$ and $0<d ! \widehat{e} \in N$. (5.7.4) $\left(x^{*}, y^{*}\right)$ is a basis of $M(R) \Rightarrow \operatorname{mag}\left(R, J, x^{*}, y^{*}\right) \leq \widehat{e}$. (5.7.5) $\left(x^{*}, y^{*}\right)$ is a basis of $M(R)$ with $1 \leq \operatorname{mag}\left(R, J, x^{*}, y^{*}\right)<\widehat{e} \Rightarrow \operatorname{mag}\left(R, J, x^{*}, y^{*}\right) \in N$ and $\operatorname{sic}\left(R, J, x^{*}, y^{*}\right) \neq 0$. (5.7.6) $\left(x^{*}, y^{*}\right)$ is a basis of $M(R)$ with $\operatorname{mag}\left(R, J, x^{*}, y^{*}\right)=$ $\widehat{e} \Rightarrow \operatorname{sic}\left(R, J, x^{*}, y^{*}\right)=0$. (5.7.7) $1<\widehat{e}<\infty \Rightarrow M(R)$ has a basis $(\widehat{x}, \widehat{y})$ with $\operatorname{mag}(R, J, \widehat{x}, \widehat{y})=\widehat{e}$. (5.7.8) If $\widehat{R}$ is the completion of $R$ then: $\widehat{e}=\infty \Leftrightarrow J \widehat{R}=y^{* d} \widehat{R}$ for some $y^{*} \in M(\widehat{R}) \backslash M(\widehat{R})^{2} \Leftrightarrow M(\widehat{R})$ has a basis $(\widehat{x}, \widehat{y})$ with $\operatorname{mag}(\widehat{R}, J \widehat{R}, \widehat{x}, \widehat{y})=$ $\infty$. (5.7.9) If $R$ is pseudogeometric then: $\widehat{e}=\infty \Leftrightarrow J=y^{* d} R$ for some $y^{*} \in$ $M(R) \backslash M(R)^{2} \Leftrightarrow M(R)$ has a basis $(\widehat{x}, \widehat{y})$ with $\operatorname{mag}(R, J, \widehat{x}, \widehat{y})=\infty$.

By a quadratic transform of $R$ we mean a local domain $R^{\prime}$, having the same quotient field as $R$, which is of the form $R\left[M(R) z^{-1}\right]_{P}$ for some $0 \neq z \in M(R)$ and some prime ideal $P$ in $R\left[M(R) z^{-1}\right]$ with $M(R) \subset P$; it is easy to see that then $R^{\prime}$ is a regular local ring of dimension 1 or 2 and it contains a unique nonzero principal ideal $J^{\prime}$ such that $J^{\prime}\left(M(R) R^{\prime}\right)^{d}=J R^{\prime}$; we call $\left(R^{\prime}, J^{\prime}\right)$ a quadratic transform of $(R, J)$; note that if $\operatorname{dim} R^{\prime}=1$ then $J^{\prime}=R^{\prime}$; cf. pages $16-36$ of $[\mathrm{AbhR}]$. Inspired by (5.1), (5.6) and (5.7), we say that $(R, J)$ is unitangent if $J \subset y^{* d} R+M(R)^{d+1}$ for some $y^{*} M(R) \backslash M(R)^{2}$, and we say that $(R, J)$ is resolved if $J=y^{* d} R$ for some $y^{*} \in M(R) \backslash M(R)^{2}$. Note that if $(R, J)$ is resolved and $\left(R^{\prime}, J^{\prime}\right)$ is any quadratic transform of $(R, J)$, then obviously $\left(R^{\prime}, J^{\prime}\right)$ is also resolved. Also note that if $R^{\prime}$ is any quadratic transform of $R$, then the canonical epimorphism $R^{\prime} \rightarrow R^{\prime} / M\left(R^{\prime}\right)$ induces an isomorphism of $\bar{R}$ onto a subfield of $R^{\prime} / M\left(R^{\prime}\right)$; we say that $R^{\prime}$ is residually rational over $R$ if the said subfield coincides with $R^{\prime} / M\left(R^{\prime}\right)$. In an obvious manner, we extend the definitions of "quadratic transform" and "resolved" to a 1 dimensional regular local ring $\widetilde{R}$ and a nonzero principal ideal $\widetilde{J}$ in it, and we note that then $\widetilde{R}$ is the only quadratic transform of itself, and $(\widetilde{R}, \widetilde{J})$ is always resolved. 
In the next proposition we shall investigate change of order and magnitude in a quadratic transform.

Proposition (5.8). If $d=0$ then for every quadratic transform $\left(R^{\prime}, J^{\prime}\right)$ of $(R, J)$ we have $J^{\prime}=R^{\prime}$. If $d \neq 0$ then there is at least one and at most $d$ quadratic transforms $\left(R^{\prime}, J^{\prime}\right)$ with $J^{\prime} \neq R^{\prime}$, and for each of them we have dim $R^{\prime}=2$ and $\operatorname{ord}_{R^{\prime}} J^{\prime} \leq$ d. If $\left(R^{\prime}, J^{\prime}\right)$ is nonunitangent, then for every quadratic transform $\left(R^{\prime}, J^{\prime}\right)$ of $(R, J)$ we have $\operatorname{ord}_{R^{\prime}} J^{\prime}<d$. If $d \neq 0$ and $(R, J)$ is unitangent, then there is a unique quadratic transform $\left(R^{\prime}, J^{\prime}\right)$ of $(R, J)$ with $J^{\prime} \neq R^{\prime}$; moreover, if also $e>1$, then for this $\left(R^{\prime}, J^{\prime}\right)$, upon letting $y^{\prime}=y / x, u^{\prime}=u / x^{d}$, and $f^{\prime}(X, Y)=f(X, X Y) / X^{d}$, we have: $y^{\prime} \in R^{\prime},\left(x, y^{\prime}\right) R^{\prime}=M\left(R^{\prime}\right), f^{\prime}(X, Y) \in R^{\prime}[X, Y], u^{\prime}=f^{\prime}\left(x, y^{\prime}\right), u^{\prime} R^{\prime}=J^{\prime}$, $R^{\prime}$ is residually rational over $R, f^{\prime}(X, Y)$ is a polyform of $u^{\prime}$ relative to $\left(R,\left(x, y^{\prime}\right)\right)$, $\operatorname{ord}_{R^{\prime} / x} J^{\prime}=D=d, \operatorname{mag}\left(R^{\prime}, J^{\prime}, x, y^{\prime}\right)=e-1$, and $\operatorname{sic}\left(R^{\prime}, J^{\prime}, x, y^{\prime}\right)=C$. [Note that in the equation about mag we are using the convention that $\infty-1=\infty$, and in view of (5.1.4) this amounts to saying that if $(R, J)$ is resolved then so is $\left(R^{\prime}, J^{\prime}\right)$; also note that if $1<e<2$ then, in view of (5.1.8), the equations about ord and mag tell us that $\operatorname{ord}_{R^{\prime}} J^{\prime}<d$.]

All the assertions except the equations about ord, mag and sic are straightforward; cf. Lemma (3.1) on page 12 of [AbhD] and the material on pages 16-36 of [AbhR]. Now, assuming $e>1$, as we have observed, $J^{\prime}=u^{\prime} R^{\prime}$ with $u^{\prime}=f^{\prime}\left(x, y^{\prime}\right)$, and clearly

$$
f^{\prime}(X, Y)=u_{0} Y^{D}+\sum_{j \in T} u_{j} X^{s(j)-j} Y^{D-j}
$$

with $u_{0} \in R^{\prime} \backslash M\left(R^{\prime}\right)$ and $u_{j} \in R^{\prime} \backslash M\left(R^{\prime}\right)$ for all $j \in T$, and hence $\operatorname{ord}_{R^{\prime} / x} J^{\prime}=$ $D=d, \operatorname{mag}\left(R^{\prime}, J^{\prime}, x, y^{\prime}\right)=e-1$, and $\operatorname{sic}\left(R^{\prime}, J^{\prime}, x, y^{\prime}\right)=C$.

In view of (5.1), as an immediate consequence of (5.7) and (5.8) we get

Corollary (5.9). Assume that $R$ is pseudogeometric, and $(R, J)$ is unresolved. Then $\widehat{e}<\infty$, and, upon letting $\mu=[\widehat{e}]$ (= the largest integer which is $\leq \widehat{e}$ ), there exists a unique sequence $\left(R_{i}, J_{i}\right)_{0 \leq i<\mu}$ with $\left(R_{0}, J_{0}\right)=(R, J)$ such that, for $1 \leq i<\mu,\left(R_{i}, J_{i}\right)$ is the unique quadratic transform of $\left(R_{i-1}, J_{i-1}\right)$ with $J_{i} \neq R_{i}$. Moreover, for this sequence we have $\operatorname{dim} R_{i}=2$ and $R_{i}$ is residually rational over $R$ and $\operatorname{ord}_{R_{i}} J_{i}=d$ for $1 \leq i<\mu$. Finally, there $i s$ at least one and at most $d$ quadratic transforms $\left(R_{\mu}, J_{\mu}\right)$ of $\left(R_{\mu-1}, J_{\mu-1}\right)$ with $J_{\mu} \neq R_{\mu}$, and for each of them we have $\operatorname{dim} R_{\mu}=2$ and $\operatorname{ord}_{R_{\mu}} J_{\mu}<d$.

As an immediate consequence of (5.9) we have the following:

Proper Local Desingularization Theorem (5.10). If $R$ is pseudogeometric, then there exists a positive integer $\mu^{\prime}$ having the property which says that if $\mu$ is any integer with $\mu \geq \mu^{\prime}$ and $\left(R_{i}, J_{i}\right)_{0 \leq i \leq \mu}$ is any sequence with $\left(R_{0}, J_{0}\right)=(R, J)$ such that $\left(R_{i}, J_{i}\right)$ is a quadratic transform of $\left(R_{i-1}, J_{i-1}\right)$ for $1 \leq i \leq \mu$, then $\left(R_{\mu}, J_{\mu}\right)$ is resolved.

In connection with the above corollary, our next step is to take care of the entire extended ideal $J R_{\mu}$.

So let $I$ be another nonzero principal ideal in $R$.

Given any quadratic transform $\left(R^{\prime}, J^{\prime}\right)$ of $(R, J)$, clearly there is a unique nonzero principal ideal $I^{\prime}$ in $R^{\prime}$ such that $J^{\prime} I^{\prime}=J I R^{\prime}$, and we call $\left(R^{\prime}, J^{\prime}, I^{\prime}\right)$ a quadratic transform of $(R, J, I)$. 
The ideals $J$ and $I$ are said to be coprime in $R$ if the ideal $J+I$ is not contained in any nonzero principal prime ideal in $R$. The ideal $I$ is said to have a normal crossing at $R$ if $I=x^{* a} y^{* b} R$ for some basis $\left(x^{*}, y^{*}\right)$ of $M(R)$ and some $(a, b) \in N \times$ $N$. We shall say that $(R, J, I)$ is resolved if $(R, J)$ is resolved and $J I$ has a normal crossing at $R$. Note that if $(R, J, I)$ is resolved and $\left(R^{\prime}, J^{\prime}, I^{\prime}\right)$ is any quadratic transform of $(R, J, I)$, then obviously $\left(R^{\prime}, J^{\prime}, I^{\prime}\right)$ is also resolved. In an obvious manner, we extend the definitions of "quadratic transform," "coprime," "normal crossing" and "resolved" to nonzero principal ideals $\widetilde{I}$ and $\widetilde{J}$ in a 1 dimensional regular local ring $\widetilde{R}$, and we note that then $\widetilde{I}$ always has a normal crossing at $\widetilde{R}$, and $(\widetilde{R}, \widetilde{J}, \widetilde{I})$ is always resolved.

Let

$$
\delta= \begin{cases}d & \text { if }(R, J) \text { is not resolved, } \\ 1 & \text { if }(R, J) \text { is resolved but }(R, J, I) \text { is not resolved, } \\ 0 & \text { if }(R, J, I) \text { is resolved. }\end{cases}
$$

We define the adjusted order ado $[R](J, I)$ by putting

$$
\operatorname{ado}[R](J, I)=\delta \text {. }
$$

Henceforth assume that $J$ and $I$ are coprime in $R$, and $I$ has a normal crossing at $R$. Note that now for any quadratic transform $\left(R^{\prime}, J^{\prime}, I^{\prime}\right)$ of $(R, J, I)$ we have that $J^{\prime}$ and $I^{\prime}$ are coprime in $R^{\prime}$, and $I^{\prime}$ has a normal crossing at $R^{\prime}$.

If $\delta=1$ then $I=I_{1}^{\dagger r(1)} \ldots I_{q}^{\dagger r(q)}$, where $1 \leq q \leq 2$ and $r(1), \ldots, r(q)$ are positive integers and $I_{1}^{\dagger}, \ldots, I_{q}^{\dagger}$ are pairwise distinct nonzero principal prime ideals in $R$ with $\operatorname{ord}_{R} I_{i}^{\dagger}=1$ for $1 \leq i \leq q$, and $J=J^{\dagger d}$, where $J^{\dagger}$ is a nonzero principal prime ideal in $R$ with $\operatorname{ord}_{R} J^{\dagger}=1$, and we let

$$
\nu=\max \left\{\operatorname{ord}_{R / J^{\dagger}} I_{i}^{\dagger}: 1 \leq i \leq q\right\} .
$$

Let

$$
\epsilon= \begin{cases}\widehat{e} & \text { if } 0 \neq \delta \neq 1 \\ \infty & \text { if } \delta=0 \\ \nu & \text { if } \delta=1\end{cases}
$$

We define the magnitude $\operatorname{mag}[R](J, I)$ by putting

$$
\operatorname{mag}[R](J, I)=\epsilon .
$$

The following lemma is easy to prove; cf. Lemma (10.3) of [AbhD].

Lemma (5.11). Assume that $\delta=1$. Then $\nu$ is a positive integer and there exists a unique sequence $\left(R_{i}, J_{i}, I_{i}\right)_{0 \leq i<\infty}$ with $\left(R_{0}, J_{0}, I_{0}\right)=(R, J, I)$ such that, for $0<$ $i<\infty,\left(R_{i}, J_{i}, I_{i}\right)$ is the only quadratic transform of $\left(R_{i-1}, J_{i-1}, I_{i-1}\right)$ for which $J_{i} \neq R_{i}$. Moreover, for this sequence we have

$$
\operatorname{ado}\left[R_{i}\right]\left(J_{i}, I_{i}\right)= \begin{cases}1 & \text { if } 0<i<\nu \\ 0 & \text { if } \nu \leq i<\infty\end{cases}
$$

and

$$
\operatorname{mag}\left[R_{i}\right]\left(J_{i}, I_{i}\right)= \begin{cases}\nu-i & \text { if } 0<i<\nu \\ \infty & \text { if } \nu \leq i<\infty\end{cases}
$$


and for $0<i<\infty$ and every quadratic transform $\left(R_{i}^{\prime}, J_{i}^{\prime}, I_{i}^{\prime}\right)$ of $\left(R_{i-1}, J_{i-1}, I_{i-1}\right)$ with $J_{i}^{\prime} \neq R_{i}^{\prime}$ we have ado $\left[R_{i}^{\prime}\right]\left(J_{i}^{\prime}, I_{i}^{\prime}\right)=0$.

As an immediate consequence of (5.10) and (5.11) we get the following:

Total Local Desingularization Theorem (5.12). If $R$ is pseudogeometric then there exists a positive integer $\mu^{\prime}$ having the property which says that: if $\mu$ is any integer with $\mu \geq \mu^{\prime}$ and $\left(R_{i}, J_{i}, I_{i-1}\right)_{0 \leq i \leq \mu}$ is any sequence with $\left(R_{0}, J_{0}, I_{0}\right)=$ $(R, J, I)$ such that $\left(R_{i}, J_{i}, I_{i}\right)$ is a quadratic transform of $\left(R_{i-1}, J_{i-1}, I_{i-1}\right)$ for $1 \leq$ $i \leq \mu$, then $\left(R_{\mu}, J_{\mu}, I_{\mu}\right)$ is resolved. [Note that we can take $I=R$ and then $\left.J_{\mu} I_{\mu}=J R_{\mu}.\right]$

\section{Section 6: Curve Desingularization}

Let $E$ be a nonsingular algebraic or arithmetic surface, and let $J$ be a nonzero (locally) principal ideal on $E$. Then by the above Theorem (5.12), there exists a nonsingular surface $E^{*}$, obtained by applying a finite sequence of quadratic transformations to $E$, such that the ideal $J E^{*}$ has only normal crossings. In other words, the ideal $J$ defines a curve on $E$, and the total transform (= the total inverse image) of this curve on $E^{*}$ has only normal crossings.

We can make this precise by using the language of models as explained on pages 155-192 of [AbhR]. Briefly, let $A$ be a field or a pseudogeometric Dedekind domain which is not a field (for example the ring of ordinary integers). Let $K$ be a finitely generated field extension of the quotient field of $A$ such that the transcendence degree of $K$ over the quotient field of $A$ is 2 or 1 according as $A$ is a field or a pseudogeometric Dedekind domain which is not a field. For any subring $B$ of $K$, let $V(B)$ be the set of all localizations $B_{P}$ of $B$ at the various prime ideals $P$ in $B$; we regard $B_{P}$ as a subring of $K$. A set $E$ of local rings with quotient field $K$ is said to be a projective model of $K / A$ if there exists a finite number of nonzero elements $x_{1}, \ldots, x_{n}$ in $K$ such that $E=\bigcup_{1 \leq i \leq n} V\left(A\left[x_{1} / x_{i}, \ldots, x_{n} / x_{i}\right]\right) ; E$ is said to be nonsingular if every local ring in $E$ is regular.

Henceforth let $E$ be a nonsingular projective model of $K / A$ (this is what we meant by a nonsingular algebraic or arithmetic surface).

By a preideal $I$ on $E$ we mean an assignment which to every $R$ in $E$ assigns an ideal $I(R)$ in $R ; I$ is nonzero means $I(R)$ is nonzero for every $R$ in $E$; $I$ is principal means $I(R)$ is principal for every $R$ in $E$; $I$ is said to be an ideal on $E$ if, for every subring $B$ of $K$ which is a finitely generated ring extension of $A$ with $V(B) \subset E$, we have $\left(\bigcap_{R \in V(B)} I(R)\right) R=I(R)$ for all $R \in V(B)$. Given preideals $J$ and $I$ on $E$, by $J I$ we denote the preideal on $E$ such that $(J I)(R)=J(R) I(R)$ for all $R$ in $E$. It follows that if $J$ and $I$ are nonzero principal ideals on $E$ then so is $J I$.

A nonzero principal ideal $I$ on $E$ is said to have only normal crossings if $I(R)$ has a normal crossing at $R$ for every $R$ in $E$ [the unit ideal $K$ is declared to have a normal crossing at $K$ ]. A nonzero principal ideal $J$ on $E$ is said to be resolved if $(R, J(R))$ is resolved for every $R$ in $E[(K, K)$ is declared to be resolved]. Nonzero principal ideals $J$ and $I$ on $E$ are said to be coprime if $J(R)$ and $I(R)$ are coprime in $R$ for every $R$ in $E$ [K and $K$ are declared to be coprime in $K$ ]. Given nonzero principal ideals $J$ and $I$ on $E$, we say that that $(E, J, I)$ is resolved if $J$ is resolved and $J I$ has only normal crossings.

By a quadratic transform of $E$ we mean a set of the form $E^{\prime}=(E \backslash\{R\}) \cup$ $\Omega(R)$, where $R$ is a 2 dimensional local ring in $E$ and $\Omega(R)$ is the set of all quadratic 
transforms of $R$; it can be seen that then $E^{\prime}$ is a nonsingular projective model of $K / A$; if $J$ is a nonzero principal ideal on $E$ then we say that $\left(E^{\prime}, J^{\prime}\right)$ is a quadratic transform of $(E, J)$, where $J^{\prime}$ is the preideal on $E^{\prime}$ such that $J^{\prime}\left(R^{\prime}\right)=J\left(R^{\prime}\right)$ for all $R^{\prime} \in E \backslash\{R\}$ and such that $\left(R^{\prime}, J^{\prime}\left(R^{\prime}\right)\right)$ is a quadratic transform of $(R, J)$ for all $R^{\prime} \in \Omega(R)$, and we note that then $J^{\prime}$ is a nonzero principal ideal on $E^{\prime}$; if $J$ and $I$ are nonzero principal ideals on $E$ then we say that $\left(E^{\prime}, J^{\prime}, I^{\prime}\right)$ is a quadratic transform of $(E, J, I)$, where $J^{\prime}$ and $I^{\prime}$ are the preideals on $E^{\prime}$ such that $J^{\prime}\left(R^{\prime}\right)=J\left(R^{\prime}\right)$ and $I^{\prime}\left(R^{\prime}\right)=I\left(R^{\prime}\right)$ for all $R^{\prime} \in E \backslash\{R\}$ and such that $\left(R^{\prime}, J^{\prime}\left(R^{\prime}\right), I^{\prime}\left(R^{\prime}\right)\right)$ is a quadratic transform of $(R, J, I)$ for all $R^{\prime} \in \Omega(R)$, and we note that then $J^{\prime}$ and $I^{\prime}$ are nonzero principal ideals on $E^{\prime}$ and $\left(E^{\prime}, J^{\prime}\right)$ is a quadratic transform of $(E, J)$. If $\mu$ is a nonnegative integer and $E_{0 \leq i \leq \mu}$ is a sequence with $E_{0}=E$ such that, for $0<i \leq \mu, E_{i}$ is a quadratic transform of $E_{i-1}$, then we say that $E_{\mu}$ is an iterated quadratic transform of $E$, and we note that then $E_{\mu}$ is a nonsingular projective model of $K / A$; if moreover $J_{i}$ is a nonzero principal ideal on $E_{i}$ with $J_{0}=J$ such that, for $0<i \leq \mu,\left(E_{i}, J_{i}\right)$ is a quadratic transform of $\left(E_{i-1}, J_{i-1}\right)$, then we say that $\left(E_{\mu}, J_{\mu}\right)$ is an iterated quadratic transform of $(E, J)$; if also $I_{i}$ is a nonzero principal ideal on $E_{i}$ with $I_{0}=I$ such that, for $0<i \leq \mu,\left(E_{i}, J_{i}, I_{i}\right)$ is a quadratic transform of $\left(E_{i-1}, J_{i-1}, I_{i-1}\right)$, then we say that $\left(E_{\mu}, J_{\mu}, I_{\mu}\right)$ is an iterated quadratic transform of $(E, J, I)$, and we note that if $J$ and $I$ are coprime then so are $J_{\mu}$ and $I_{\mu}$.

Henceforth let $J$ and $I$ be nonzero principal ideals on $E$ such that $J$ and $I$ are coprime and $I$ has only normal crossings.

It is easy to see that then $(R, J(R), I(R))$ is resolved for all except a finite number of 2 dimensional local rings $R$ in $E$. Therefore by (5.12) we get the following:

Theorem (6.1). There exist nonzero principal ideals $J^{*}$ and $I^{*}$ on a nonsingular projective model $E^{*}$ of $K / A$ such that $\left(E^{*}, J^{*}, I^{*}\right)$ is resolved and $\left(E^{*}, J^{*}, I^{*}\right)$ is an iterated quadratic transform of $(E, J, I)$. [Note that we can take $I(R)=R$ for all $R \in E$; then we have $J^{*} I^{*}=J E^{*}$, and hence $J E^{*}$ has only normal crossings].

\section{REFERENCES}

[AbhR] S. S. Abhyankar, Resolution of Singularities of Embedded Algebraic Surfaces, Academic Press, New York, 1966. MR 36:164

[AbhD] S. S. Abhyankar, Desingularization of Plane Curves, Proceedings of Symposia in Pure Mathematics 40 (1983), 1-45. MR 85d:14024

[AbhW] S. S. Abhyankar, Weighted Expansions for Canonical Desingularization, Springer Verlag, New York, 1982. MR 84m:14013

[AbhG] S. S. Abhyankar, Good Points of a Hypersurface, Advances in Mathematics 68 (1988), 87-256. MR 89e: 14012

[Bhas] Bhaskaracharya, Beejaganit, 1150.

[Mats] H. Matsumura, Commutative Algebra, Second Edition, Benjamin, Reading, 1980. MR 82i:13003

[Naga] M. Nagata, Local Rings, Interscience, New York, 1962. MR 27:5790

[ZarS] O. Zariski and P. Samuel, Commutative Algebra II, Van Nostrand, Princeton, 1960. MR 22:11006

Department of Mathematics, Purdue University, West Lafayette, Indiana 47907

E-mail address: ram@cs.purdue.edu 